Editorial

\title{
Transcatheter Aortic Valve Implantation in Singapore: Reflecting on the First Decade
}

Edgar LW Tay, ${ }^{1}$ MRCP (UK), MMed (Int Med), FAMS

Transcatheter aortic valve implantation (TAVI) has markedly changed the way aortic valve stenosis is treated. It is 18 years since the first patient was treated with TAVI for severe aortic stenosis. ${ }^{1}$ The original concept of TAVI was simple: insert a stent-like device by creating an opening in the obstructed aortic valve, and then suture a valve within the stent to ensure aortic competence. The technical achievement of this feat, however, took the time and ingenuity of both engineers and interventional cardiologists/surgeons with contributions from the imaging team, anaesthesiologists and nurses to pull it off. This close collaboration led to the birth of heart valve teams, and the initial success of TAVI has spurred further optimisation in procedural techniques, devices, improved patient selection and better team experience. These advances have culminated in the safe treatment of most patients with degenerative aortic valve stenosis by TAVI today.

Although the history of TAVI in Singapore occurred in tandem with the rest of the world, its journey is a unique one. Asian patients are different in many ways. First, there is a culture of reluctance to undergo invasive surgical treatment, especially in the elderly. A local study had found that a large number of patients declined conventional surgery even after it was recommended to them by their physicians. ${ }^{2}$ Consequently, a significantly less invasive procedure such as TAVI could potentially offer these patients a more palatable option.

Second, Asian patients tend to have smaller body sizes and the larger size of TAVI devices in the early days resulted in a high incidence of vascular injury when they were introduced into the femoral artery. Additionally, apical bleeding of the left ventricle was seen when a transapical approach was used. However, with time, these problems were resolved with the development of devices that had smaller profiles and improved operator experience.

Third, the reimbursement landscape of TAVI continues to pose a challenge. The procedure is costly and is currently not fully subsidised by the state. Elderly patients have a tendency to trivialise their symptoms to avoid placing further emotional and financial strain on family members when they are asked to consider TAVI. In the last decade, most patients who underwent TAVI had received financial assistance to defray the costs of the procedure from various sources that included philanthropic societies, health and medical insurance, personal savings and hospital endowment funds and funds pooled from fundraising efforts.

The first TAVI was performed in the National Heart Centre Singapore in February 2009 via transfemoral access in a 77-year-old patient who presented with high surgical risks. ${ }^{3}$ Subsequently, it was offered to patients of the National University Hospital from November 2010. To date, $>650$ patients were treated in 3 hospitals (including a third centre in Mount Elizabeth Hospital). Each year, approximately 100 TAVI procedures are performed. With a population of around 4 million residents (citizens and permanent residents) in Singapore, this translates into 25 cases in 1,000,000 residents. This rate is still low compared to countries where TAVI is fully subsidised by the state. However, the number is likely to increase in coming years. This is attributed to an ageing population, growing evidence from trials that support the efficacy and safety of TAVI in low-risk patients (2 recent landmark trials ${ }^{4,5}$ had shown non-inferiority in 1 while the other demonstrated superiority of TAVI over conventional surgery) and likelihood of subsidies that will be offered by the state to defray the costs of the procedure.

In their article entitled "Impact of chronic kidney disease on outcomes in transcatheter aortic valve implantation" in this issue of the Annals, Yap et $\mathrm{al}^{6}$ described a sizeable cohort of patients who had undergone TAVI in Singapore. By illustrating the effect of pre-existing renal function and the changes that followed TAVI, they highlighted that advanced

${ }^{1}$ Department of Cardiology, National University Heart Centre Singapore, Singapore

Address for Correspondence: Dr Edgar Tay Lik Wui, Department of Cardiology, National University Heart Centre Singapore, NUHS Tower Block, Level 9, 1E Kent Ridge Road Singapore 119228.

Email: edgar_tay@nuhs.edu.sg 
kidney disease impacts on mortality and procedural outcomes such as postoperative paravalvular aortic regurgitation. The authors should be lauded for their contribution in a field where local data is still emerging.

The systematic collection of data in a local TAVI registry is important since it promotes improvement in patient care. Specifically, it provides a good understanding of patients who would benefit from TAVI and those who may develop higher rates of postoperative complications. It also allows information to be shared among colleagues and to be used for educational purposes within institutions and at the national level. Although there were some differences in methodologies, TAVI outcomes ${ }^{6,7}$ in the 3 local centres were comparable to those reported by top TAVI centres from around the world.

With continued innovation, the future of TAVI remains bright for patients in Singapore. Several classes of newly developed TAVI devices are already being used by clinicians to treat patients. They include cerebral protection devices to reduce strokes, newer valve designs to reduce paravalvular leak and vascular injury and new vascular closure devices to reduce vascular complications.

Nevertheless, several challenges need to be addressed in the near term. The first relates to the durability of TAVI valves. Since these valves may be implanted in younger, lower-risk individuals, consideration must be paid to the potential of a repeat intervention after the valves have deteriorated. Although repeat TAVIalso known as a valve-in-valve procedure-is possible, longer-terms studies are needed. In some patients, the impact of cardiac electrical conduction disturbancesuch as left bundle branch block or pacemakers - and increased difficulty of coronary re-access for future coronary angiography and angioplasty have not been definitively addressed.

The second challenge is the high costs of TAVI and a likely increase in its volume from a rapidly ageing population. Although several studies have demonstrated the cost efficacy of TAVI over conventional surgery, marked differences in the cost and delivery of health services across different countries must be factored into consideration. Studies on the cost effectiveness of TAVI against surgical aortic valve replacement in Singapore are needed to inform various funding agencies on the amount of subsidies that is required in future. Additionally, local studies such as the one by Yap et $\mathrm{al}^{6}$ may help to identify subgroups of patients who are most likely to benefit from TAVI.
The third challenge relates to training of surgeons who are skilled in surgical aortic valve replacement. Traditionally, aortic valve replacement is a vital skill that most cardiac surgeons acquire in the course of their training. With the increasing number of TAVI procedures that are being performed, the potential reduction in the number of surgical cases may impact on the learning curve of cardiac surgery trainees, and the longer-term impact is also not known. Additionally, consideration to make TAVI part of cardiac surgical training is still ongoing.

In the last decade, the growth and development of TAVI in Singapore has been truly fascinating. The initial work of pioneers in TAVI had developed quickly into a mature and well accepted medical therapy. Currently, more complex procedures are being undertaken. ${ }^{8}$ In the next decade, there is much potential for improvement. With heart valve teams at its core, patients with aortic stenosis in Singapore can look forward to better clinical outcomes from treatment with TAVI.

\section{REFERENCES}

1. Cribier A, Eltchaninoff H, Bash A, Borenstein N, Tron C, Bauer F, et al. Percutaneous transcatheter implantation of an aortic valve prosthesis for calcific aortic stenosis: first human case description. Circulation 2002;106:3006-8.

2. Tay ELW, Lew PS, Poh KK, Saclolo R, Chia BL, Yeo TC, et al. Demographics of severe valvular aortic stenosis in Singapore. Singapore Med J 2013;54:36-9.

3. Chiam PT, Koh TH, Chao VT, Lee CY, See Tho VY, Tan SY, et al. Percutaneous transcatheter aortic valve replacement: first transfemoral implant in Asia. Singapore Med J 2009;50:534-7.

4. Popma JJ, Deeb GM, Yakubov SJ, Mumtaz M, Gada H, O’Hair D, et al. Transcatheter aortic-valve replacement with a self-expanding valve in low-risk patients. N Engl J Med 2019;380:1706-15.

5. Mack MJ, Leon MB, Thourani VH, Makkar R, Kodali SK, Russo M, et al. Transcatheter aortic-valve replacement with a balloon-expandable valve in low-risk patients. N Engl J Med 2019;380:1695-705.

6. Yap JJL, Tay JCK, Ewe SH, Thiagarajan N, Chia SY, Amanullah MR, et al. Impact of chronic kidney disease on outcomes in transcatheter aortic valve implantation. Ann Acad Med Singapore 2020;49:273-84.

7. Chew N, Hon JKF, Yip JWL, Chan SP, Poh KK, Kong WKF, et al. Mid-term study of transcatheter aortic valve implantation in an Asian population with severe aortic stenosis: two-year Valve Academic Research Consortium-2 outcomes. Singapore Med J 2017;58:543-50.

8. Tay ELW, Hon JKF, Yip JWL, Teoh KLK, Poh KK, Ang SBL, et al. Combined transcatheter therapy of aortic stenosis and thoracic aortic aneurysm. Ann Acad Med Singapore 2014;43:279-81. 